\title{
New insights into naevoid melanomas: a clinicopathological reassessment
}

DOI:

10.1111/his.13317

\section{Document Version}

Accepted author manuscript

Link to publication record in Manchester Research Explorer

\section{Citation for published version (APA):}

Cook, M., Massi, D., Blokx, W. A. M., van den Oord, J., Koljenovic, S., De Giorgi, V., Kissin, E., Grant, M., Mandal, A., Gremel, G., Gaudy, C., Viros Usandizaga, A., Dhomen, N., Khosrotehrani, K., Marais, R., Green, A., \& Mihm Jr, M. C. (2017). New insights into naevoid melanomas: a clinicopathological reassessment. Histopathology. https://doi.org/10.1111/his.13317

Published in:

Histopathology

\section{Citing this paper}

Please note that where the full-text provided on Manchester Research Explorer is the Author Accepted Manuscript or Proof version this may differ from the final Published version. If citing, it is advised that you check and use the publisher's definitive version.

\section{General rights}

Copyright and moral rights for the publications made accessible in the Research Explorer are retained by the authors and/or other copyright owners and it is a condition of accessing publications that users recognise and abide by the legal requirements associated with these rights.

\section{Takedown policy}

If you believe that this document breaches copyright please refer to the University of Manchester's Takedown Procedures [http://man.ac.uk/04Y6Bo] or contact uml.scholarlycommunications@manchester.ac.uk providing relevant details, so we can investigate your claim.

\section{OPEN ACCESS}




\section{New insights into naevoid melanomas: a clinico-pathological reassessment}

Martin G Cook ${ }^{1,2,3,4}$, Daniela Massi ${ }^{4,5}$, Willeke A.M. Blokx ${ }^{4,6}$, Joost Van den Oord ${ }^{4,7}$, Senada Koljenović ${ }^{4,8}$, Vincenzo De Giorgi ${ }^{10}$, Eleanor Kissin ${ }^{11}$, Megan Grant ${ }^{2}$, Amit Mandal $^{2}$, Gabriela Gremel ${ }^{2}$, Caroline Gaudy ${ }^{2}$, Amaya Viros $^{2}$, Nathalie Dhomen ${ }^{2}$, Kiarash Khosrotehrani ${ }^{13,14}$, Richard Marais ${ }^{2}$, Adele C Green ${ }^{\star 2,12}$, Martin C Mihm Jr ${ }^{\star 4,9}$

[1] Histopathology, Royal Surrey County Hospital, Egerton Road, Guildford GU2 $7 X X$, UK

[2] Molecular Oncology Group, CRUK Manchester Institute, University of Manchester, Wilmslow Road, Manchester M20 4BX, UK

[3] Division of Clinical Medicine, University of Surrey, Guildford, Surrey, GU2 7XH, UK

[4] Member of EORTC Melanoma Group Pathology Working Group

[5] Division of Pathological Anatomy, Department of Surgery and Translational Medicine, University of Florence, Italy

[6] Department of Pathology, Radboud University Medical Center, Nijmegen, The Netherlands

[7] Translational Cell and Tissue Research, Department of Imaging and Pathology, University of Leuven (KU Leuven), Leuven, Belgium

[8] Department of Pathology, Erasmus University Medical Center, Rotterdam, The Netherlands

[9] Brigham and Women's Hospital, Boston, MA 02115 USA 
[10] Department of Dermatology, University of Florence, Italy

[11] Eleanor Kissin, Department of Plastic Surgery, St George's Hospital, London, UK

[12] QIMR Berghofer Medical Research Institute, Brisbane, Queensland, Australia [13] Department of Dermatology, Princess Alexandra Hospital, Brisbane, Queensland, Australia

[14] The University of Queensland, UQ Diamantina Institute, Translational Research Institute, Brisbane, Queensland, Australia

*These authors contributed equally to the paper

Running title: Naevoid melanoma

${ }^{*}$ Correspondence to: Prof M. G. Cook, Consultant Histopathologist, Histopathology, Royal Surrey County Hospital, Egerton Road, Guildford GU2 7XX, UK. Email: m.cook@nhs.net

No conflicts of interest.

Word Count: 2155 


\section{Abstract}

Aims: Because the term 'naevoid melanoma' has variable clinical and pathologic interpretations, we aimed to clarify the features of melanomas referred to as naevoid.

Methods and Results: A review was undertaken of 102 melanomas diagnosed histopathologically as naevoid melanomas and ascertained by European Organisation for Research and Treatment of Cancer Melanoma Group Subcommittee pathologists from their records. We found these could be classified morphologically into 3 groups. Thirteen melanomas were overlying genuine naevi and were therefore excluded. Of the 89 melanomas considered to be naevoid, 11 presented clinically as exophytic papillomatous nodules with little junctional component and composed of small atypical cells showing numerous mitoses and no change with depth; we termed these "papillomatous naevoid" melanomas. The other 78 were flat or only slightly raised and had a superficial spreading melanoma (SSM)like component with maturation to a small cell, but still atypical, dermal component; we termed these "maturing naevoid" melanomas. We showed that papillomatous and maturing naevoid melanomas also have differing immunochemical profiles.

Preliminary clinical follow-up suggested different outcomes for these two naevoid melanoma types.

Conclusions: Melanomas that have been classified as naevoid melanomas comprise two types with distinct clinical, histopathologic and immunohistochemical features that may also be prognostically significant.

Key words: Maturing naevoid melanoma, papillomatous naevoid melanoma, immunohistochemistry, small cell melanoma, paradoxical maturation. 


\section{Introduction}

Although the term "naevoid melanoma" could potentially describe a melanoma which resembles any variant of melanocytic naevus, the term is usually restricted to relatively uncommon melanomas resembling common intradermal or compound naevi in which the dermal component predominantly comprises small naevoid cells. Some of these lesions can be easily mistaken for benign naevi and may only be recognised when a recurrence occurs ${ }^{(1,2)}$. The existence of such melanomas was noted originally by Levene ${ }^{(3)}$ and by others subsequently ${ }^{(4,5)}$. The previous term 'minimal deviation melanoma' ${ }^{(6)}$ possibly included naevoid melanomas and they have also been referred to as small cell melanoma ${ }^{(7,8)}$. Ruhoy et al ${ }^{(9)}$ in describing a melanoma with paradoxical maturation appeared to be referring to the same lesion that Blessing et al ${ }^{(8)}$ termed 'small cell naevoid melanoma'. Such practice has resulted in confusion among pathologists and clinicians treating patients with potentially different lesions but with the same diagnostic label. The aim of this study was to re-evaluate the histological and clinical features of naevoid melanomas in order to clarify whether lesions so classified include one or more types of melanoma.

\section{Materials and Methods}

The study was approved by the Royal Surrey County Hospital Ethics Committee (07/Q190913). All cases diagnosed as naevoid melanoma in the period 2004 to 2016 were retrieved from the files of members of the Pathology Subcommittee of the European Organisation for Research and Treatment of Cancer Melanoma Group (EORTC MG) and the consultation files of one of us (MGC). Cases were included if histological sections were available and patient's age, sex and anatomical site of lesion known. Naevoid lesions resembling spitz naevi as well as other lesions such 
as deep penetrating naevi and blue naevi were regarded as separate entities and were specifically excluded.

All eligible cases underwent histological review of the features in the Royal College Minimum Data Set ${ }^{(10)}$ for melanomas on original H\&E stained sections. Immunohistochemical staining of further sections of all cases of both subtypes was carried out to descriptively characterise the tumours. The 64 maturing naevoid melanomas where blocks were available were further stained with CD44 and markers that might help explain the phenomenon of maturation. For example reticulin staining was carried out as a measure of stroma around naevus or melanoma cells, while P16 and RRM2 stains were chosen to reflect senescence. Other markers employed on 24 of the 64 maturing naevoid melanomas were p21, ECadherin, N-Cadherin, WHSC1/NSD2 and 5-hmC. Negative controls substituting normal serum for primary antibodies were employed throughout. The antibodies HMB-45, MIB-1, P16, E-Cadherin and CD44 were obtained from DAKO (Cambridgeshire), for N-Cadherin from Leica Bio-systems and for RRM2 from Abcam. Staining for p21 was performed in Rotterdam (by SK), that for WHSC1/NSD2 in Leuven (JvdO), and that for 5-hmC in Boston by courtesy of Dr Christine Lian ${ }^{(11)}$. All cases were assessed systematically by a single pathologist (MGC) and independently reviewed by other members of the EORTC MG Pathology Group using a multi-head microscope. Any disagreement was resolved in discussion.

Molecular analysis was performed of 11 cases where paraffin tissue was available. DNA from micro-dissected formalin-fixed, paraffin-embedded (FFPE) material was isolated using GeneRead DNA FFPE kits (Qiagen, Hilden, Germany) according to 
manufacturer's instructions. PCR-based target enrichment and next-generation sequencing was performed as previously described ${ }^{(12)}$.

\section{Results}

Between 2004 and 2016, 102 cases were retrieved from the participating pathologists' files. On initial review, 13 melanomas associated with a benign preexisting naevus were excluded, leaving 89 naevoid melanoma cases for study.

\section{$\underline{\text { Patient features }}$}

The average age of patients with papillomatous naevoid melanoma was 40 years (range 12-89 years), significantly younger than 56 years (range 16-89 years) for maturing naevoid melanoma patients ( $p=0.001)$, and males comprised $40 \%$ vs $56 \%$ respectively. For the papillomatous subtype, $40 \%$ were on the head and $60 \%$ on the trunk, while of the maturing subtype, $8 \%$ were on the head; $44 \%$ on the trunk and $48 \%$ on the limbs.

\section{$\underline{\text { Histology }}$}

Detailed histology showed the 89 cases separated into 2 groups with distinctly different patterns of growth (Table 1). One group of 11 cases showed an exophytic papillomatous configuration (Figs 1-3; 4), herein termed "papillomatous naevoid" melanoma. This group resembling predominantly intradermal naevi had a mean thickness of $2.0 \mathrm{~mm}$ (range 1.3 - 3.7). The epidermis showed prolongations into the underlying lesion with only a scanty or focal junctional proliferation of melanocytes

(Fig 1) and occasionally a complete absence of junctional melanocytes. The epidermal prolongations appeared to divide the underlying melanocytes into lobules. The constituent melanocytes appeared small mainly due to a lack of cytoplasm but the nuclei were larger than those of a dermal naevus and appeared hyperchromatic 
and often angulated and facetted against each other (Fig 2). Mitoses were always present (mean count: 5.9 per $\mathrm{mm}^{2 ;}$ range 4 to 7 ) and the proliferation marker MIB-1 showed a band of cells with a high proportion of positivity adjacent to the epidermal prolongations (Fig 3). The melanocytes did not show maturation but in 2 cases the underlying dermis included benign naevus cells (Fig 4), allowing a direct comparison with the naevoid melanoma cells. Apart from differences in size, shape and chromicity the tumour cells were surrounded by a less conspicuous collagenous stroma and reticulin framework in groups of 3-5 cells whereas the collagen in preexisting naevi was more prominent and reticulin surrounded smaller groups of cells. The second larger group comprised 78 cases of largely flat or shallow dome-shaped lesions distinguished by their histology as superficially invasive melanoma predominantly of SSM type but showing a change in cell type in the superficial dermis (Table 1). We termed these "maturing naevoid" melanomas. The mean thickness was $0.9 \mathrm{~mm}$ (range $0.4-3.0$ ) and mitoses were few (mean mitotic rate $0.7 / \mathrm{mm}^{2}$; range $\left.0-1\right)$. The evidence of malignancy in this group comprised the histological features of atypia and tumour architecture that indicated infiltration of the dermis. Specifically, the maturing variants had a disorderly junctional proliferation of severely atypical melanocytes (Fig 5) with similar cells extending into the superficial dermis but to a variable depth before showing a change in cell type and arrangement (Fig 6). With depth the cells become smaller, with less cytoplasm, round hyperchromatic nuclei and they show an irregular nested pattern. Light melanin pigmentation was usually evident in the cytoplasm (Table 1).

The essential feature of this maturing naevoid group was the change in cell type at varying levels in the papillary dermis or superficial reticular dermis. The small naevoid cells either showed a sheet-like arrangement (Fig 6) with nesting more 
deeply or showed an immediate nesting pattern surrounded by dense collagen (Fig 7). The nests tended to be larger in the deeper part of the lesion, in the same pattern described by others ${ }^{(7)}$.

Although the deeper cells could be said to be naevoid, in the 13 cases where a true pre-existing naevus was present, the distinction was clear (Fig 8). This was due to the larger hyperchromatic nuclei and their grouping together with reticulin surrounding irregular groups within the larger aggregates in contrast to the smaller naevus cells with reticulin around smaller groups of cells. Of the 78 cases of maturing naevoid melanoma there were 19 where this process did not affect the full width of the lesion. The maturation ranged from focal, affecting $10-20 \%$ of the lesion (5 cases) to nearly complete, affecting $80-90 \%$ of the lesion ( 1 case).

$\underline{\text { Immunohistochemistry }}$

Immunohistochemical staining in the papillomatous naevoid melanomas (Table 2) indicated active proliferation and showed characteristics associated with malignancy. In addition CD44 showed uniform strong membranous staining. RRM2 staining was strong throughout.

In contrast, immunohistochemistry of all 78 maturing naevoid melanomas showed a diminution in proliferative activity in the deeper component, and other markers also showed features tending towards senescence (Fig 9). In cases showing lack of uniformity, such immunochemical findings applied only to the part with features suggesting maturation. In the maturing naevoid melanomas stained with RRM2, the maturing part of the tumour was no longer represented in 7 cases but the superficial invasive component was positive in all. In the 13 cases where both superficial and maturing components remained available for assessment, 3 showed weak or negative staining in the deep maturing components but the rest remained positive. 


\section{$\underline{\text { Molecular analysis }}$}

We analysed 1-3 micro-dissected areas of 11 available cases: 3 papillomatous and 8 maturing naevoid melanomas. Specifically, we performed targeted sequencing of $B R A F$ and NRAS genes using next-generation sequencing technology. All 3 papillomatous naevoid cases carried a hotspot mutation in the NRAS gene (seen consistently across 1, 2 and 3 micro-dissected areas for each case respectively) but were wild type for BRAF (Table 2). Of the 8 maturing naevoid melanomas, 2 carried a BRAF hotspot mutation (consistently observed across 2 and 4 micro-dissected areas respectively), 4 carried an NRAS hotspot mutation (consistently observed across up to 3 micro-dissected areas), and 2 cases were wild type for both $B R A F$ and NRAS (Table 2).

\section{Preliminary follow-up}

Early follow-up data were available for $90 \%$ patients with the papillomatous type (average follow-up, 3.3 years) and $64 \%$ of those with the maturing type (average follow-up, 2 years). In-transit or lymph node metastases had occurred in $33 \%$ of patients with papillomatous naevoid melanomas (with measured thicknesses $<1.0 \mathrm{~mm}, 2.1 \mathrm{~mm}$ and $3.7 \mathrm{~mm}$ respectively) but no disease progression was seen in those with maturing naevoid melanomas (including 2 with measured thickness $3.0 \mathrm{~mm}$ and $3.5 \mathrm{~mm}$ respectively).

\section{Discussion}

In this study of atypical melanocytic tumours with features of benign melanocytic naevi and melanoma, we confirmed that most cases could be classified as melanoma on the basis of atypia, disorderly growth pattern or mitoses. These could be separated into two groups, one resembling a raised predominantly intradermal nevus that we termed "papillomatous naevoid" and a second resembling a 
compound naevus with junctional dysplasia that we termed "maturing naevoid". The less frequent papillomatous naevoid group has been previously described by

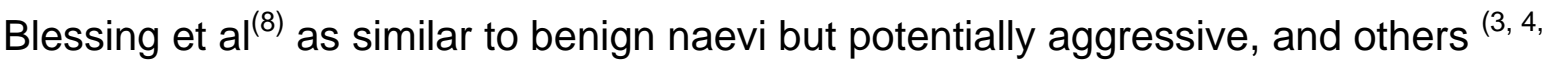
${ }^{13)}$ who also noted similar prognosis to other melanomas, with recurrence and metastatic rate of $50 \%$ reported ${ }^{(2)}$ for papillomatous naevoid melanomas ${ }^{(1)}$. Apart from Blessing et al ${ }^{(8)}$, LeBoit and Massi ${ }^{(14),}$ Ruhoy et al ${ }^{(9)}$ and Kossard and Wilkinson ${ }^{(7)}$, other authors appear not to have recognised the maturing naevoid group as a distinct variant of melanoma. Blessing et al ${ }^{(8)}$ referred to them as small cell variants of naevoid melanoma that may be less aggressive than other melanomas. Ruhoy et al $^{(9)}$ described them as melanoma with paradoxical maturation without mention of difference in behaviour while others ${ }^{(14)}$ recognised papillomatous and non-papillomatous naevoid melanomas corresponding to our findings but again did not note any difference in behaviour between the two morphological types. Kossard and Wilkinson ${ }^{(7)}$ described a small cell naevoid melanoma with a lentiginous epidermal component and features which appear similar to some of our maturing naevoid melanomas. They noted a nested dermal component with fibrosis and they suspected a less aggressive behaviour.

On the other hand, Yélamos et al $^{(15)}$ described naevoid melanoma as a heterogeneous group: those with high grade atypia having worse prognosis. Our observations of two distinct types of naevoid melanoma were supported by immunochemical differences. All papillomatous variants showed raised Ki67/MIB1 counts, consistent with its marked proliferative activity, while the maturing naevoid cells showed staining with $\mathrm{P} 16$ and $5-\mathrm{hmC}$ consistent with being intermediate between melanoma and true naevus cells ${ }^{(12)}$ presumably suggesting progression towards senescence. Indeed the immunohistochemical data raise the question 
whether the maturing naevoid melanoma is appropriately classified. RRM2 was present in the superficial dermal part supporting their classification as invasive melanomas and senescence not being the sole basis of observed changes ${ }^{(16)}$. For the moment, maturing naevoid lesions appear appropriately classified as melanomas but the intralesional changes suggest acquisition of a less aggressive behaviour and our preliminary follow-up data support this notion. Finally, the molecular findings that all 3 of the papillomatous naevoid cases carried a hotspot mutation in the NRAS gene but were wild type for $B R A F$, was a striking result even in so small a cohort, given that $B R A F$ is the most commonly mutated melanoma driver gene. The observations for the 8 maturing naevoid melanomas, largely recapitulated previous findings that NRAS and BRAF hotspot mutations are generally mutually exclusive (in one case, a non-hotspot BRAF mutation was observed in 2 of the 3 micro-dissected areas of an NRAS mutated maturing naevoid melanoma), although the relatively more common occurrence of NRAS mutations compared to $B R A F$ mutations was notable. A larger cohort of cases would need to be analysed at the molecular level to assess whether these types of lesions are more commonly driven by RAS.

Whilst it is well known that many tumours including melanoma show heterogeneity, it is less accepted that tumours having acquired properties of invasion and proliferation can reverse those properties. Whether the observations are correctly interpreted as maturation ${ }^{(9)}$ and if this is a response to cell signalling, suppressed mutations or an effect produced by the microenvironment, are unknown, and we will seek to address these questions in further studies. 


\section{Acknowledgements}

The work was supported by Cancer Research UK (Ref C5759/A20971). Meetings of the EORTC MG Pathology Group were supported by the EORTC MG.

\section{Author contributions}

Conceptualised and conducted the study: MGC, DM, WB, JvdO, SK, MM

Collected and managed the study data: MGC, ACG, KK, VdG, EK, MG, AM, GG, CG, AV, ND, RM

Data interpretation: MGC, ACG, KK, DM, WB, JvdO, SK, MM, VdG, EK, MG, AM, GG, CG, AV, ND, RM

Drafted the manuscript: MGC, ACG, DM, WB, JvdO, SK, MM, VdG, EK, MG, AM, GG, CG, AV, ND , KK, RM

All authors approved the final manuscript.

\section{Abbreviations}

HMB-45, human melanoma black 45; MIB-1, methylation-inhibited binding protein 1; CD44, Cluster of Differentiation 44; WHSC1, Wolf-Hirschhorn Syndrome Candidate 1; NSD2, Nuclear Set Domain-Containing 2; 5-hmC, 5-Hydroxymethylcytosine; RRM2, RNA Recognition Motif 2. 


\section{References}

1. McNutt NS. "Triggered trap": nevoid malignant melanoma. Seminars in diagnostic pathology. 1998;15(3):203-9.

2. Zembowicz A, McCusker M, Chiarelli C, Dei Tos AP, Granter SR, Calonje E, et al.

Morphological analysis of nevoid melanoma: a study of 20 cases with a review of the literature. The American Journal of dermatopathology. 2001;23(3):167-75.

3. Levene A. On the histological diagnosis and prognosis of malignant melanoma. Journal of clinical pathology. 1980;33(2):101-24.

4. Schmoeckel C, Wagner-Grosser G, Braun-Falco O. [Clinical diagnosis of early malignant melanomas]. Hautarzt. 1985;36(10):558-62.

5. Blessing K, Evans AT, al-Nafussi A. Verrucous naevoid and keratotic malignant melanoma: a clinico-pathological study of 20 cases. Histopathology. 1993;23(5):453-8.

6. Podnos YD, Jimenez JC, Zainabadi K, Jakowatz JG, Barr RJ. Minimal deviation melanoma. Cancer treatment reviews. 2002;28(5):219-21.

7. Kossard S, Wilkinson B. Small cell (naevoid) melanoma: a clinicopathologic study of 131 cases. The Australasian journal of dermatology. 1997;38 Suppl 1:S54-8.

8. Blessing K, Grant JJ, Sanders DS, Kennedy MM, Husain A, Coburn P. Small cell malignant melanoma: a variant of naevoid melanoma. Clinicopathological features and histological differential diagnosis. Journal of clinical pathology. 2000;53(8):591-5.

9. Ruhoy SM, Prieto VG, Eliason SL, Grichnik JM, Burchette JL, Jr., Shea CR. Malignant melanoma with paradoxical maturation. The American journal of surgical pathology. 2000;24(12):1600-14.

10. Pathologists TRCo. Cancer datasets and tissue pathways: Skin. 2014:https://www.rcpath.org/profession/publications/cancer-datasets.html.

11. Lee JJ, Cook M, Mihm MC, Xu S, Zhan Q, Wang TJ, et al. Loss of the epigenetic mark, 5Hydroxymethylcytosine, correlates with small cell/nevoid subpopulations and assists in microstaging of human melanoma. Oncotarget. 2015;6(35):37995-8004.

12. Girotti MR, Gremel G, Lee R, Galvani E, Rothwell D, Viros A, et al. Application of Sequencing, Liquid Biopsies, and Patient-Derived Xenografts for Personalized Medicine in Melanoma. Cancer Discovery. 2016;6(3):286-99.

13. Divan A H, and Alexander, J. L. Naevoid Melanoma. Clin Lab Med. 2011;31:243 - 53.

14. LeBoit P, and Massi, G. . Histological Diagnosis of Nevi and Melanoma. Springer. 2014;2nd Edition:467-80.

15. Yelamos O, Busam KJ, Lee C, Meldi Sholl L, Amin SM, Merkel EA, et al. Morphologic clues and utility of fluorescence in situ hybridization for the diagnosis of nevoid melanoma. Journal of cutaneous pathology. 2015;42(11):796-806.

16. Aird KM, Zhang G, Li H, Tu Z, Bitler BG, Garipov A, et al. Suppression of nucleotide metabolism underlies the establishment and maintenance of oncogene-induced senescence. Cell reports. 2013;3(4):1252-65. 
Table 1. Microscopic features of two subtypes of naevoid melanomas

\section{Papillomatous}

Raised/papillomatous nodule

Flat or shallow dome

Frequent epidermal strands causing segmentation

Epidermis without segmentation

Little intraepidermal melanocytic proliferation

Intraepidermal severe atypia amounting to melanoma

Hyperchromatic angulated cells

Epithelioid upper, small cells in deep part

Little cytoplasm

More cytoplasm in upper part, less in deep

Sheets showing little variation

Changes in cell type from top to deep nests becoming larger deeper

Mitoses numerous at periphery of nodules

Mitoses few and superficial only 
Table 2. Immunohistochemistry and molecular analysis of two subtypes of naevoid melanomas

\begin{tabular}{|c|c|c|}
\hline Marker & Papillomatous & Maturing \\
\hline HMB-45 & Positive & Superficial positive, deep negative \\
\hline MIB1/Ki67 & High count, notably periphery of compartments & Negative deep \\
\hline P16 & Negative throughout & Negative becoming positive deeper \\
\hline WHSC1 (NSD2) & Positive throughout & Superficial positive, deep negative \\
\hline 5-hmC & Negative & Variable positive \\
\hline P21 & Positive throughout & Positive superficial, scanty or negative deep \\
\hline Reticulin & Around large groups of dermal cells & Around small groups but not individual cells \\
\hline E-Cadherin & Negative & Negative becoming positive deep \\
\hline $\mathrm{N}$-Cadherin & Variable positive & Superficial variable positive, weaker deep \\
\hline CD44 & Strong positive & Negative to variable weak \\
\hline RRM2 & Positive throughout & $\begin{array}{l}\text { Superficial dermal positive } \\
\text { Deeper component } \\
\text { Variable to negative }\end{array}$ \\
\hline BRAF hotspot & $0 \%$ positive $(0 / 3)$ & $25 \%(2 / 8)$ \\
\hline NRAS hotspot & $100 \%$ positive $(3 / 3)$ & $50 \%(4 / 8)$ \\
\hline
\end{tabular}


Figs 1 Typical exophytic papillomatous naevoid melanoma with epidermal strands compartmentalising the tumour cells and showing little junctional proliferation.

Fig 2 Same papillomatous naevoid melanoma as Fig 1 showing hyperchromatic angulated nuclei centrally and mitoses evident peripherally.

Fig 3 Same papillomatous naevoid melanoma as Figs 1, 2 with high MIB-1 count at the periphery of the lobules.

Fig 4 Hyperchromatic naevoid melanocytes with little cytoplasm adjacent to true benign naevus cells.

Fig 5 Maturing naevoid melanoma. Disordely large atypical melanoma cells (upper) contrasting with smaller round cells with little cytoplasm (deep).

Fig 6 Another maturing naevoid melanoma showing the change from larger epitheloid cells in the papillary dermis to smaller hyperchromatic cells in the deeper dermis.

Fig 7 A different maturing naevoid melanoma showing a change from epithelioid pleomorphic nested melanocytes in the superficial dermis to, in the deeper part, smaller but still atypical cells arranged in nests by dense collagen.

Fig 8 A maturing naevoid melanoma in which small atypical melanocytes in superficial dermis are arranged in nests surrounded by dense collagen. A true benign naevus is present at the deep aspect of this melanoma.

Fig 9 An example of the immunohistochemical staining P21 is seen positive in the junctional and superfical dermal component of another maturing naevoid melanoma that is largely negative in the deeper small cell component. 